\title{
MDM2 and CDK4 amplifications are rare events in salivary duct carcinomas
}

\author{
Inga Grünewald ${ }^{1}$, Marcel Trautmann ${ }^{1}$, Alina Busch², Larissa Bauer ${ }^{1}$, Sebastian \\ Huss $^{1}$, Petra Schweinshaupt ${ }^{3}$, Claudia Vollbrecht ${ }^{3,4}$, Margarete Odenthal ${ }^{3}$, \\ Alexander Quaas ${ }^{3}$, Reinhard Büttner ${ }^{3}$, Moritz F. Meyer ${ }^{5}$, Dirk Beutner ${ }^{5}$, Karl-Bernd \\ Hüttenbrink ${ }^{5}$, Eva Wardelmann ${ }^{1}$, Markus Stenner ${ }^{2}$, Wolfgang Hartmann ${ }^{1}$ \\ ${ }^{1}$ Department of Pathology, University Hospital Muenster, Muenster, Germany \\ ${ }^{2}$ Department of Otorhinolaryngology, Head and Neck Surgery, University Hospital Muenster, Muenster, Germany \\ ${ }^{3}$ Institute of Pathology, University Hospital Cologne, Cologne, Germany \\ ${ }^{4}$ Current address: Institute of Pathology, Charité University Hospital Berlin, Berlin, Germany \\ ${ }^{5}$ Department of Otorhinolaryngology, Head and Neck Surgery, University Hospital Cologne, Cologne, Germany \\ Correspondence to: Inga Grünewald, email: inga.gruenewald@ukmuenster.de \\ Keywords: salivary gland carcinoma, p53, MDM2, CDK4, HMGA2 \\ Received: February 15, $2016 \quad$ Accepted: September 12, 2016 \\ Published: September 20, 2016
}

\section{ABSTRACT}

Salivary duct carcinoma (SDC) is an aggressive adenocarcinoma of the salivary glands associated with poor clinical outcome. SDCs are known to carry TP53 mutations in about $\mathbf{5 0 \%}$, however, only little is known about alternative pathogenic mechanisms within the p53 regulatory network. Particularly, data on alterations of the oncogenes MDM2 and CDK4 located in the chromosomal region 12q13-15 are limited in SDC, while genomic rearrangements of the adjacent HMGA2 gene locus are well documented in subsets of SDCs. We here analyzed the mutational status of the TP53 gene, genomic amplification of MDM2, CDK4 and HMGA2 rearrangement/amplification as well as protein expression of TP53 (p53), MDM2 and CDK4 in 51 de novo and ex pleomorphic adenoma SDCs.

25 of 51 cases were found to carry TP53 mutations, associated with extreme positive immunohistochemical p53 staining levels in 13 cases. Three out of 51 tumors had an MDM2 amplification, one of them coinciding with a CDK4 amplification and two with a HMGA2 rearrangement/amplification. Two of the MDM2 amplifications occurred in the setting of a TP53 mutation. Two out of 51 cases showed a CDK4 amplification, one synchronously being MDM2 amplified and the other one displaying concurrent low copy number increases of both, MDM2 and HMGA2.

In summary, we here show that subgroups of SDCs display genomic amplifications of MDM2 and/or CDK4, partly in association with TP53 mutations and rearrangement/amplification of HMGA2. Further research is necessary to clarify the role of chromosomal region 12q13-15 alterations in SDC tumorigenesis and their potential prognostic and therapeutic relevance.

\section{INTRODUCTION}

Salivary duct carcinoma (SDC) is an aggressive adenocarcinoma of the salivary glands, most commonly involving the parotid gland. It is one of the most aggressive salivary gland malignancies, most frequently associated with the occurrence of early distant metastasis and poor prognosis [1]. In the past few years increasing knowledge on recurrent genetic alterations of SDC evolved. In recent studies high percentages of TP53 mutations were detected, involving around $50 \%$ of cases of de novo and ex pleomorphic adenoma SDCs [2,3]. However, there is a large subset of TP53 wildtype (WT) tumors which might harbor alternative alterations in the p53 regulatory network.

For decades, p53 has been a well-known tumor suppressor that is mutated or functionally inactivated 
in large subsets of human cancers [4]. Physiologically, transcriptional activity and stability of p53 are negatively regulated by the ubiquitin ligase MDM2, involving at least two mechanisms: a) direct blockage of the p53 transactivation domain and b) ubiquitylation-induced proteasomal degradation. Overexpression of MDM2, as found in many human tumors, is therefore capable to functionally impair p53. Inhibition of the MDM2-p53 interaction may therefore restore p53 activity and might offer opportunities for a targeted cancer therapy in tumors characterized by MDM2 overexpression [5]. In this setting, a study performed on MDM2-amplified welldifferentiated or dedifferentiated liposarcomas (LS), which are consistently characterized by a high-level genomic amplification (frequently $>15-30$ copies as clusters) of sequences derived from chromosomal region $12 \mathrm{q} 13-15$ comprising the oncogenes MDM2 and CDK4, showed that treatment with the MDM2 antagonist RG7112 activates the p53 pathway and decreases cell proliferation [6]. Most frequently, MDM2 protein deregulation occurs in tumors that retain wildtype TP53, but MDM2 alterations have also been described in subsets of TP53 mutated tumors $[4,6]$. It has been shown before that genomic instability affecting the chromosomal region 12q13-15 occurs in subsets of salivary gland carcinomas [7]. Apart from these findings, results from $M D M 2$ transgenic mice developing mammary gland tumors suggest a crucial role for MDM2 in epithelial tumors of glandular differentiation [8].

In LS, the 12q13-15 amplicon usually, but not always, shows a co-amplification of the cell cycle regulator $C D K 4$ together with MDM2 [9]. It has been shown that the small subgroup of $M D M 2+/ C D K 4$ - LS shows favorable prognostic features compared to $M D M 2+/ C D K 4+\mathrm{LS}$ [10]. Knowledge on the CDK4 amplification status therefore provides genomic information on the structural characteristics of the amplicon with $M D M 2$ being located at $12 \mathrm{q} 15$ and $C D K 4$ at $12 \mathrm{q} 13.3-12$ and it might add further information on an independent oncogenic mechanism apart from p53 dysfunction. Since CDK4 is the key regulator of the G1-S cell-cycle transition and drives cell-cycle progression, CDK4 inhibitors might offer new strategies for a targeted cancer therapy $[11,12]$.

Another gene in chromosomal region 12q13-15 frequently subject to structural alterations is $H M G A 2$, encoding a high-mobility group protein. Chromosomal breaks of the HMGA2 locus have been described in several benign mesenchymal tumors including lipomas and uterine leiomyomas [13, 14], and $H M G A 2$ amplification was shown in several soft tissue malignancies including liposarcomas where it is almost always co-amplified with MDM2 [9, 15]. In salivary gland tumors, rearrangements/ amplifications of $H M G A 2$ are well known in subsets of pleomorphic adenomas (PA) $[14,16]$. Carcinomas ex PA have been reported to generally retain $H M G A 2$ rearrangements along with further gene alterations in tumor progression making $H M G A 2$ a potential marker for SDCs arising in PA [16].
Only very little is known about the role of MDM2 or CDK4 in SDC tumorigenesis. The major aim of this study therefore was to systematically evaluate the involvement of MDM2 and CDK4 alterations in SDC and to put them in context with $H M G A 2$ alterations known in SDC. We here report on the rare occurrence of MDM2 and CDK4 amplifications in a large collection of these aggressive salivary neoplasms showing a heterogeneous distribution among TP53 wildtype tumors and those carrying a TP53 mutation.

\section{RESULTS}

51 SDC cases were analyzed for TP53 mutational status, $M D M 2, C D K 4$ and $H M G A 2$ genomic amplification as well as $H M G A 2$ rearrangement and p53, MDM2 and CDK4 protein expression. The clinicopathological characteristics of these 51 patients are summarized in Table 1, and the results of the mutation screen, FISH and immunohistochemical analyses are displayed in Figure 1. In Figure 2, images of immunohistochemical stainings and FISH analyses are shown exemplarily for cases M117 (Figure 2A), K210 (Figure 2B) and M52 (Figure 2C).

TP53 mutations were detected in 25 of these 51 cases (Table 2). In 8 cases, the TP53 mutational status could not be evaluated due to insufficient DNA content. Immunohistochemistry for p53 showed an extreme positive (EP) staining result in 13 cases, all of these cases displaying a TP53 missense point mutation except for one case in which the TP53 mutational status could not be evaluated due to minor DNA quality. In 8 cases with various TP53 mutations, an extreme negative (EN) p53 immunohistochemical staining was detected and 5 cases with TP53 mutations showed a non-extreme (NE) staining level for p53. An extreme negative (EN) p53 staining was detected in 8 cases without TP53 mutation and in 5 further cases in which TP53 mutational status could not be evaluated.

Three out of the total 51 cases displayed an MDM2 amplification, one case showed an MDM2 grey-zone amplification in FISH analyses. Two cases with MDM2 amplification strongly stained for MDM2, one MDM2 amplified case and the grey-zone amplified case showed low MDM2 immunohistochemical staining levels. Two of the MDM2 amplified cases synchronously carried TP53 mutations. All MDM2 amplifications and the MDM2 greyzone amplification occurred in de novo SDCs, none was detected in an SDC ex pleomorphic adenoma.

In two cases, we detected a $C D K 4$ amplification, one coinciding with an $M D M 2$ amplification and one in the setting of an $M D M 2$ grey-zone amplification. Nine cases showed a $C D K 4$ grey-zone amplification, one of these cases in the setting of an MDM2 amplification. However, the immunohistochemical CDK4 staining was negative in all cases. 
Table 1: Clinical data of the patients included in the study

\begin{tabular}{|c|c|}
\hline Patients' characteristics & $\mathbf{N}(\%)$ \\
\hline Patients & 51 \\
\hline Male & $38(74.5 \%)$ \\
\hline Female & $13(25.5 \%)$ \\
\hline \multicolumn{2}{|l|}{ Age (years) } \\
\hline Mean $\pm \mathrm{SD}$ & $66.2 \pm 13.0$ \\
\hline Median & 68 \\
\hline Minimum/Maximum & $36 / 90$ \\
\hline \multicolumn{2}{|l|}{ Histology } \\
\hline $\mathrm{SDC}$ de novo & $39(76.5 \%)$ \\
\hline SDC ex pleomorphic adenoma & $12(23.5 \%)$ \\
\hline \multicolumn{2}{|l|}{ Resection margins } \\
\hline R0 & $25(49.0 \%)$ \\
\hline R1 & $17(33.3 \%)$ \\
\hline $\mathrm{R} 2$ & $1(2.0 \%)$ \\
\hline $\mathrm{Rx}$ & $8(15.7 \%)$ \\
\hline \multicolumn{2}{|l|}{ pT-stage } \\
\hline $\mathrm{pTx}$ & $1(2.0 \%)$ \\
\hline pT1 & $12(23.5 \%)$ \\
\hline pT2 & $5(9.8 \%)$ \\
\hline pT3 & $16(31.4 \%)$ \\
\hline pT4a & $16(31.4 \%)$ \\
\hline pT4b & $1(2.0 \%)$ \\
\hline \multicolumn{2}{|l|}{ pN-stage } \\
\hline $\mathrm{pNx}$ & $2(3.9 \%)$ \\
\hline $\mathrm{pN} 0$ & $10(19.6 \%)$ \\
\hline $\mathrm{pN} 1$ & $8(15.7 \%)$ \\
\hline $\mathrm{pN} 2$ & $30(58.8 \%)$ \\
\hline $\mathrm{pN} 3$ & $1(2.0 \%)$ \\
\hline \multicolumn{2}{|l|}{ Extracapsular spread } \\
\hline Unknown & $23(45.1 \%)$ \\
\hline Yes & $15(29.4 \%)$ \\
\hline No & $13(25.5 \%)$ \\
\hline \multicolumn{2}{|l|}{ cM-stage } \\
\hline $\mathrm{cMx}$ & $12(23.6 \%)$ \\
\hline $\mathrm{cM} 0$ & $30(58.8 \%)$ \\
\hline cM1 & $9(17.6 \%)$ \\
\hline
\end{tabular}




\begin{tabular}{ll}
\hline Patients' characteristics & $\mathbf{N}(\mathbf{\%})$ \\
\hline Lymphangiosis & $15(29.4 \%)$ \\
Unknown & $22(43.1 \%)$ \\
Yes & $14(27.5 \%)$ \\
No & \\
Hemangiosis & $18(35.3 \%)$ \\
Unknown & $14(27.5 \%)$ \\
Yes & $19(37.3 \%)$ \\
No & \\
Perineural invasion & $19(37.3 \%)$ \\
Unknown & $22(43.1 \%)$ \\
Yes & $10(19.6 \%)$ \\
No & \\
Type of parotidectomy & $1(2.0 \%)$ \\
Unknown & $4(7.8 \%)$ \\
Lateral & $24(47.1 \%)$ \\
Total & $20(39.2 \%)$ \\
Radical & $2(3.9 \%)$ \\
Subtotal & \\
Neck dissection & \\
Yes & $49(96.1 \%)$ \\
No & $2(3.9 \%)$ \\
\hline
\end{tabular}

HMGA2 FISH could be analyzed in 42 out of 51 cases. 5 cases showed rearrangement and/or amplification of $H M G A 2$, among these 3 de novo and 2 ex PA SDCs. Two of the 5 cases displayed HMGA2 rearrangement and amplification, both occurring in association with an $M D M 2$ amplification; the third $M D M 2$ (and $C D K 4$ ) amplified case displayed no HMGA2 alteration. One of the $H M G A 2$ rearrangements occurred in the setting of a $C D K 4$ grey-zone amplification, the two further $H M G A 2$ rearrangements were detected in cases without $M D M 2$ and/or $C D K 4$ amplification. One CDK4 amplified tumor with grey-zone $M D M 2$ amplification showed only a low $H M G A 2$ copy number increase.

The 5-year overall survival (OS) in our collection of SDCs was 39.6\% (Figure 3A). TP53 mutated cases showed a tendency to a worse 5 -year OS $(20.5 \%$ with TP53 mutation vs. $53.3 \%$ without TP53 mutation) but statistical significance was not reached $(\mathrm{p}=0.267)$ (Figure 3B). No statistically significant differences in 5-year OS were detected for $M D M 2$ and/or CDK4 amplified/greyzone amplified subgroups.

\section{DISCUSSION}

Recent genomic profiling studies confirmed a high frequency of TP53 mutations in de novo and ex PA SDCs $[2,3]$, affecting about $50 \%$ of the tumors, pointing to an outstanding relevance of the p53 tumor suppressing network in the pathogenesis of these rare but highly aggressive tumors. With particular regard to the remaining $50 \%$ of SDCs harboring wildtype sequences for TP53, the major aim of this study was to elucidate the role of another key player within the p53 regulatory network, i.e. the ubiquitin ligase MDM2, in SDC. Due to the colocalization of $M D M 2$ with the cell cycle regulator $C D K 4$ in the chromosomal region 12q13-15 and a documented proneness of this region to genomic alterations, these regulators of cell growth and fate are frequently coamplified in some human malignancies including a subset of soft tissue tumors [9]. Apart from providing details on the characteristics of the amplicon, knowledge of the CDK4 amplification status might add further information on an independent oncogenic mechanism apart from p53 dysfunction, opening therapeutic options. Involvement 


\begin{tabular}{|c|c|c|c|c|c|c|c|c|}
\hline $\begin{array}{l}\frac{\bar{d}}{\mathbf{2}} \\
\mathbf{\xi} \\
\mathbf{z}\end{array}$ & 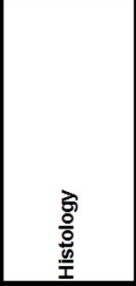 & 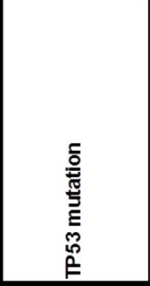 & 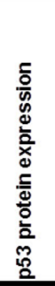 & 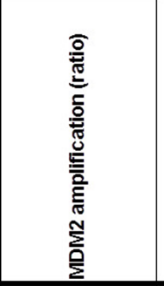 & 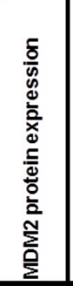 & 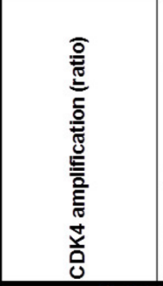 & 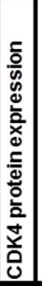 & 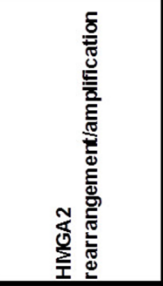 \\
\hline M7 & SDC de novo & no & NE & no & low & $\begin{array}{c}\text { grey-zone (1.77; } \\
\text { microclusters) }\end{array}$ & no & no \\
\hline M14 & SDC de novo & missense & NE & no & no & $\begin{array}{c}\text { grey-zone (1.39; } \\
\text { microclusters) }\end{array}$ & no & no \\
\hline M16 & SDC de novo & missense & EP & no & no & no & no & no \\
\hline M18 & SDC de novo & missense & NE & no & low & no & no & no \\
\hline M20 & SDC de novo & missense & EP & no & low & no & no & no \\
\hline M25 & SDC de novo & nonsense & NE & no & no & no & no & no \\
\hline M32 & SDC de novo & $\begin{array}{l}\text { missense AND } \\
\text { frameshift_del }\end{array}$ & EN & no & low & no & no & RA (unbalanced) \\
\hline M37 & SDC de novo & no & NE & no & low & no & no & no \\
\hline M39 & SDC de novo & nonsense & EN & no & low & no & no & no \\
\hline M45 & SDC de novo & missense & EP & no & no & no & no & no \\
\hline M48 & SDC de novo & no & EN & no & no & no & no & n.a. \\
\hline M52 & SDC de novo & nonsense & EN & yes (4.92) & strong & no & no & RA and ampl. \\
\hline M55 & SDC de novo & frameshift_del & EN & no & low & no & no & no \\
\hline M61 & SDC exPA & no & $\mathrm{NE}$ & no & no & no & no & n.a. \\
\hline M62 & $S D C$ exPA & no & $\mathrm{EN}$ & no & no & no & no & no \\
\hline M64 & $S D C$ exPA & no & EN & no & no & no & no & no \\
\hline M67 & $S D C$ exPA & missense & EP & no & low & no & no & RA (unbalanced) \\
\hline M72 & $S D C$ exPA & missense & EP & no & low & no & no & no \\
\hline M78 & SDC de novo & no & $\mathrm{NE}$ & no & low & no & no & no \\
\hline M84 & SDC exPA & no & $\mathrm{NE}$ & no & low & no & no & no \\
\hline M93 & SDC exPA & missense & EP & no & no & no & no & no \\
\hline M96 & SDC de novo & no & EN & no & no & no & no & no \\
\hline M102 & SDC de novo & no & $\mathrm{NE}$ & no & low & no & no & no \\
\hline M104 & SDC de novo & no & NE & no & low & no & no & no \\
\hline M106 & SDC de novo & nonsense & NE & no & low & no & no & no \\
\hline M107 & SDC de novo & no & NE & no & no & \begin{tabular}{|c|} 
no \\
\end{tabular} & no & no \\
\hline M108 & $S D C$ exPA & missense & EP & no & low & $\begin{array}{c}\text { grey-zone (1.47; } \\
\text { microclusters) }\end{array}$ & no & no \\
\hline M110 & SDC de novo & $\begin{array}{c}\text { missense AND } \\
\text { missense }\end{array}$ & EP & no & no & 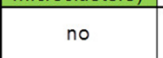 & no & no \\
\hline M114 & SDC exPA & missense & EP & no & no & no & no & no \\
\hline M117 & SDC de novo & missense & EP & yes (4.28) & strong & \begin{tabular}{|l|} 
yes $(5.91)$ \\
\end{tabular} & no & no \\
\hline $\mathrm{K} 3$ & SDC de novo & no & EN & yes (3.72) & low & grey-zone (1.96) & no & RA and ampl. \\
\hline K6 & SDC de novo & $\begin{array}{c}\text { missense AND } \\
\text { missense }\end{array}$ & EP & no & low & no & no & no \\
\hline K24 & SDC de novo & n.a. & EP & no & low & no & no & no \\
\hline K96 & SDC de novo & n.a. & EN & no & no & $\begin{array}{c}\text { grey-zone (1.39; } \\
\text { microclusters) }\end{array}$ & no & no \\
\hline K103 & SDC de novo & $\begin{array}{c}\text { missense AND } \\
\text { missense }\end{array}$ & EN & no & no & $\begin{array}{c}\text { grey-zone (1.50; } \\
\text { microclusters) }\end{array}$ & no & no \\
\hline K124 & SDC exPA & missense & EP & no & no & grey-zone (1.98) & no & RA \\
\hline K134 & SDC de novo & no & EN & no & no & no & no & no \\
\hline K135 & SDC de novo & n.a. & EN & no & no & no & no & n.a. \\
\hline K141 & SDC de novo & no & NE & no & low & no & no & n.a. \\
\hline K146 & SDC de novo & n.a. & NE & no & low & no & no & no \\
\hline K162 & SDC de novo & no & EN & no & no & no & no & n.a. \\
\hline K173 & SDC de novo & nonsense & EN & no & no & no & no & no \\
\hline K189 & $\mathrm{SDC} \operatorname{exPA}$ & missense & $\mathrm{NE}$ & no & no & no & no & n.a. \\
\hline K204 & SDC de novo & no & $\mathrm{NE}$ & no & low & no & no & no \\
\hline K207 & SDC de novo & frameshift_ins & EN & \begin{tabular}{|c|} 
no \\
\end{tabular} & no & no & no & no \\
\hline K210 & SDC de novo & no & EN & $\begin{array}{c}\text { grey-zone (1.77; } \\
\text { microclusters) }\end{array}$ & low & yes (2.52) & no & $\begin{array}{l}\text { low copy number } \\
\text { increase }(3.35)\end{array}$ \\
\hline K222 & SDC de novo & n.a. & EN & no & no & no & no & n.a. \\
\hline K240 & SDC de novo & nonsense & EN & no & low & $\begin{array}{c}\text { grey-zone (1.45; } \\
\text { microclusters) }\end{array}$ & no & no \\
\hline K242 & SDC de novo & n.a. & EN & no & low & no & no & no \\
\hline K258 & SDC de novo & n.a. & EN & no & low & grey-zone (1.85) & no & n.a. \\
\hline K277 & $\mathrm{SDC} \operatorname{exPA}$ & n.a. & $\mathrm{NE}$ & no & low & no & no & n.a. \\
\hline
\end{tabular}

Figure 1: Results of immunohistochemical stainings for p53, MDM2 and CDK4 and fluorescence in-situ hybridization analyses for $M D M 2, C D K 4$ and $H M G A 2$, displayed for each case (PA: pleomorphic adenoma; n.a.: not available; EP: extreme positive; EN: extreme negative; NE: non-extreme, RA: rearrangement; ampl.: amplification). 
of the chromosomal region $12 q 13-15$ in salivary gland tumorigenesis has been documented before with the $H M G A 2$ gene showing rearrangements and genomic amplification in subsets of PA and SDCs ex PA [16]. Since $H M G A 2$ furthermore represents an almost constant partner of $M D M 2$ in the 12q13-15 amplicon known in other tumors, we complemented our analysis with the analysis of $H M G A 2$ to get further insight into the structure of the amplicon [9].

We detected MDM2 amplifications in 3 cases, interestingly all classified as de novo SDCs. Two of these MDM2 amplified cases showed a concurrent rearrangement/co-amplification of $H M G A 2$. According to Bahrami et al. [16], who proposed alterations of $H M G A 2$ as a marker for SDCs ex PA, it could be hypothesized if these two cases might also have arisen in totally obscured PA, however, there was no evidence for a pre-existing PA, neither by histopathology nor with respect to clinical history. One of the $M D M 2$ amplified SDCs harbored no $H M G A 2$ alteration while 3 SDCs with $H M G A 2$ rearrangement did not show an MDM2 amplification, implying that alterations of these two genes are obviously not strictly connected in SDCs. Thus, MDM2 amplification appears to be a genomic alteration at least partly independent from $H M G A 2$ status and particularly from pathogenesis (de novo vs. ex PA) in SDC. Since only two of the cases harboring an MDM2 amplification revealed a strong immunohistochemical staining for MDM2, immunohistochemical staining alone appears not to be a reliable marker of $M D M 2$ amplification in SDC. Interestingly, two of the MDM2 amplified cases synchronously carried TP53 mutations, while one MDM2 amplification occurred in a TP53wildtype setting. In another TP53-wildtype tumor a grey-zone $M D M 2$ amplification was detected. A very recent study on $37 \mathrm{SDCs}$ revealed one $M D M 2$ amplified case, also in the setting of a TP53 mutation [2]. When focusing on the MDM2-p53 regulatory connection, mechanistically, tumors with functionally inactivating mutations in TP53 cannot be expected to substantially gain in oncogenic potential due to a concomitant amplification of $M D M 2$ since it usually acts as an oncogenic factor by
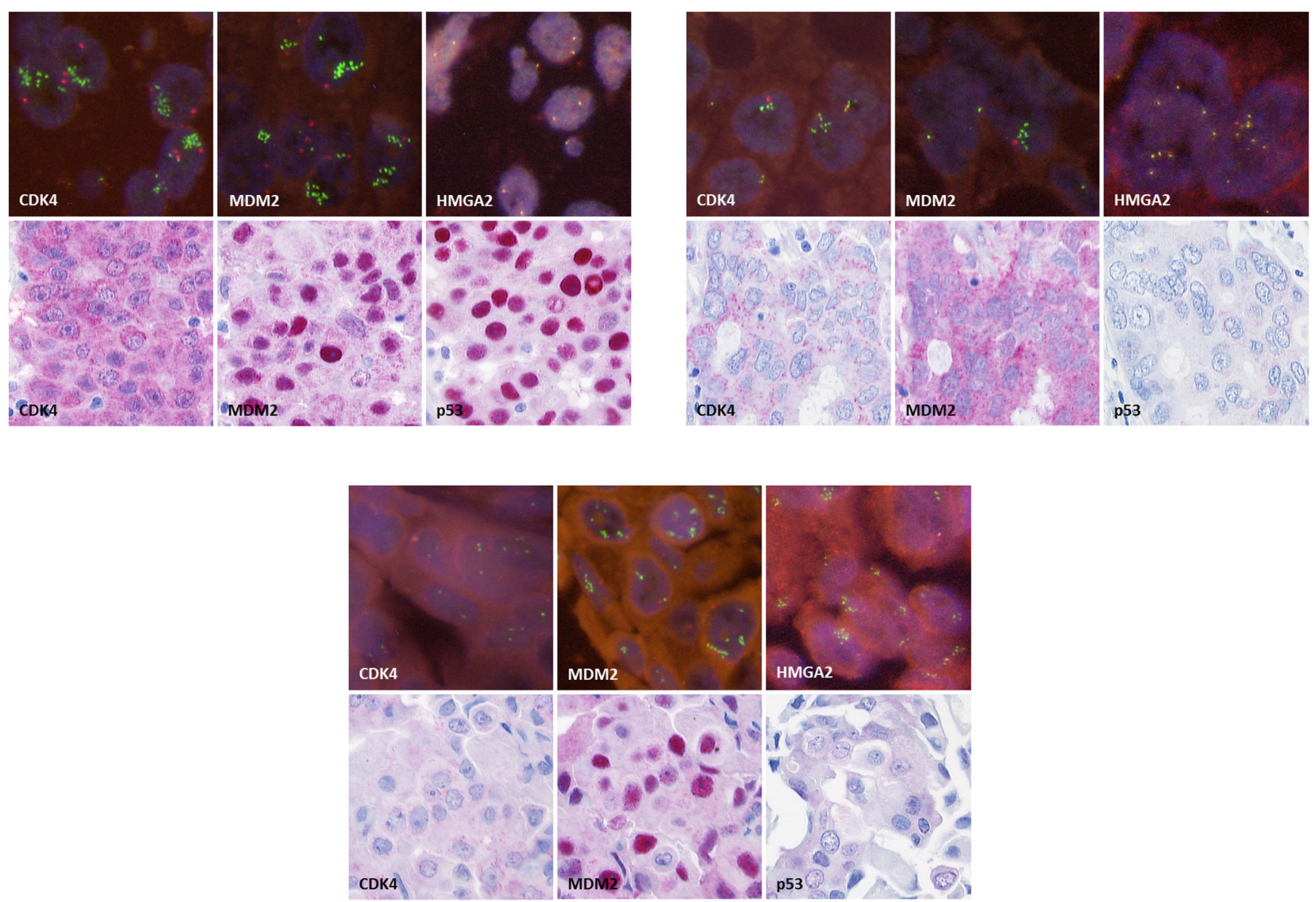

Figure 2: Immunohistochemical stainings for CDK4, MDM2 and p53 (original magnification 250x) and fluorescence in-situ hybridization for $C D K 4, M D M 2$ and $H M G A 2$ (original magnification 630x), exemplarily for case M117 A., case K210 B. and case M52 C. 
Table 2: List of TP53 mutations detected in the analysis of 51 SDCs

\begin{tabular}{|c|c|}
\hline No. & TP53 mutation \\
\hline M14 & Exon 8 p.Pro278Arg $($ c. $833 \mathrm{C}>\mathrm{G})$ \\
\hline M16 & Exon 5 p.Tyr163His $(\mathrm{c} .487 \mathrm{~T}>\mathrm{C})$ \\
\hline M18 & Exon 4 p.Pro89Leu (c.266C>T) \\
\hline M20 & Exon 7 p.Gly245Ser (c.733G $>$ A) \\
\hline M25 & Exon 10 p.Arg $342 *($ c. $1024 C>T)$ \\
\hline M32 & $\begin{array}{l}\text { Exon } 4 \text { p.Pro } 75 \text { Leu (c.224C>T); Exon } 6 \text { p.Arg209fs } \\
\text { (c.626_627delGA) }\end{array}$ \\
\hline M39 & Exon 6 p.Gln $192 *($ c. $574 C>T)$ \\
\hline M45 & Exon 5 p.Asn131Tyr (c.391A>T) \\
\hline M52 & Exon 8 p.Arg306* (c.916C >T) \\
\hline M55 & Exon 6 p.Arg209fs (c.626_627delGA) \\
\hline M67 & Exon 8 p.Phe270Cys $($ c.809T $>$ G) \\
\hline M72 & Exon 5 p.Lys132Glu (c.394A>G) \\
\hline M93 & Exon 6 p.Tyr220Cys (c.659A>G) \\
\hline M106 & Exon 9 p.Gln $331 *(c .991 C>T)$ \\
\hline M108 & Exon 5 p.Arg 175 His (c. $524 \mathrm{G}>\mathrm{A})$ \\
\hline M110 & Exon 7 p.Ile232Met (c.696C >G); p.Ser241Phe $(\mathrm{c} 722 \mathrm{C}>\mathrm{T})$ \\
\hline M114 & Exon 5 p.His168Arg $($ c.503A>G) \\
\hline M117 & Exon 5 p.Ser127Phe $($ c. $380 \mathrm{C}>\mathrm{T})$ \\
\hline K6 & $\begin{array}{l}\text { Exon } 7 \text { p.Gly } 245 \text { Val (c. } 734 G>T) \text {, Exon } 10 \text { p.Gly360Ala } \\
(\text { c. } 1079 \mathrm{G}>\text { C) }\end{array}$ \\
\hline K103 & Exon 8 p.Arg273Leu (c.818G $>$ T), p.Phe270Ser $($ c.809T $>$ C) \\
\hline K124 & Exon 7 p.Asn239Ser (c.716A>G) \\
\hline K173 & Exon 4 p.Tyr103* (c.309C $>$ A) \\
\hline K189 & Exon 6 p.His214Arg (c.641A>G) \\
\hline K207 & Exon 8 p.Val274fs (c.819_820insT) \\
\hline K240 & Exon 4 p.Gln $100^{*}($ c. $298 \mathrm{C}>\mathrm{T})$ \\
\hline
\end{tabular}

downregulation of wildtype p53 protein. In contrast, in tumors harboring particular TP53 mutations and retaining their wildtype TP53 allele, MDM2 amplification might contribute to oncogenesis by additionally disrupting the remaining functional p53 levels. Additionally, several reports argue in favor of an oncogenic role for $M D M 2$ independently from the p53 status $[8,17,18]$. In any case, based on our findings, in a small subset of SDCs, MDM2 amplification obviously may serve as an alternative mechanism leading to dysregulation of the p53 network, thereby offering an option for a targeted MDM2-directed therapy [6]. With respect to documented activation steps of MDM2 through AKT1 in salivary acinar cells and mammary epithelium [19, 20], MDM2 amplification might represent an alternative or additional mechanism in deregulating the p53 network besides well-known and pathogenetically relevant genetic alterations of the PI3K/ AKT signaling pathway in SDC [3].

The immunohistochemical staining for p53 partly correlated with the TP53 mutational status: With $48 \%$ of TP53 mutated cases and none of the TP53 wildtype cases showing an extreme positive (EP) staining for $\mathrm{p} 53$ protein expression, EP p53 staining reliably went along with a missense TP53 mutation. $32 \%$ of TP53 mutated cases showed an extreme negative (EN) p53 staining and 20\% of TP53 mutated cases a non-extreme (NE) p53 staining. In contrast, cases without TP53 mutation displayed an EN p53 staining result in $44 \%$ and a NE staining in 
$56 \%$. So, in summary, in case of an EN or a NE p53 staining, a reliable prediction of TP53 mutational status is not possible in SDCs whereas an EP p53 expression is strongly suggestive of an underlying TP53 missense mutation.

Interestingly, TP53 mutated cases showed a tendency to a worse 5-year OS (Figure 3B), pointing to a prognostic relevance of a TP53 mutation in SDC.

Out of the MDM2 amplified SDCs identified in this study, two tumors showed a concomitant $C D K 4$ amplification, one a grey-zone $C D K 4$ amplification and one lacked an amplification of $C D K 4$. This finding is in agreement with genomic data from a subset of liposarcomas, which frequently show a variable amplification of sequences of the chromosomal region 12q13-15. Independently from a copy number change of $M D M 2$, eight tumors showed a $C D K 4$ greyzone amplification (one coinciding with a $H M G A 2$ rearrangement), pointing to a CDK4-mediated cellcycle dysregulation in SDC tumorigenesis. CDK4 inhibitors might therefore offer new strategies for targeted therapeutic approaches in SDCs as recently discussed for several entities [11, 12]. Interestingly, none of the CDK4 amplified or grey-zone amplified cases showed a positive immunohistochemical staining for CDK4 (with solid staining result of the positive control), so immunohistochemical CDK4 staining obviously is not reliably suitable for the detection of $C D K 4$ amplified SDC. Anyway, lack of immunohistochemical CDK4 protein detection does not exclude a pathogenic role of the CDK4 oncogene and may be due to tight protein regulation beyond the threshold of staining sensitivity. Contrasting
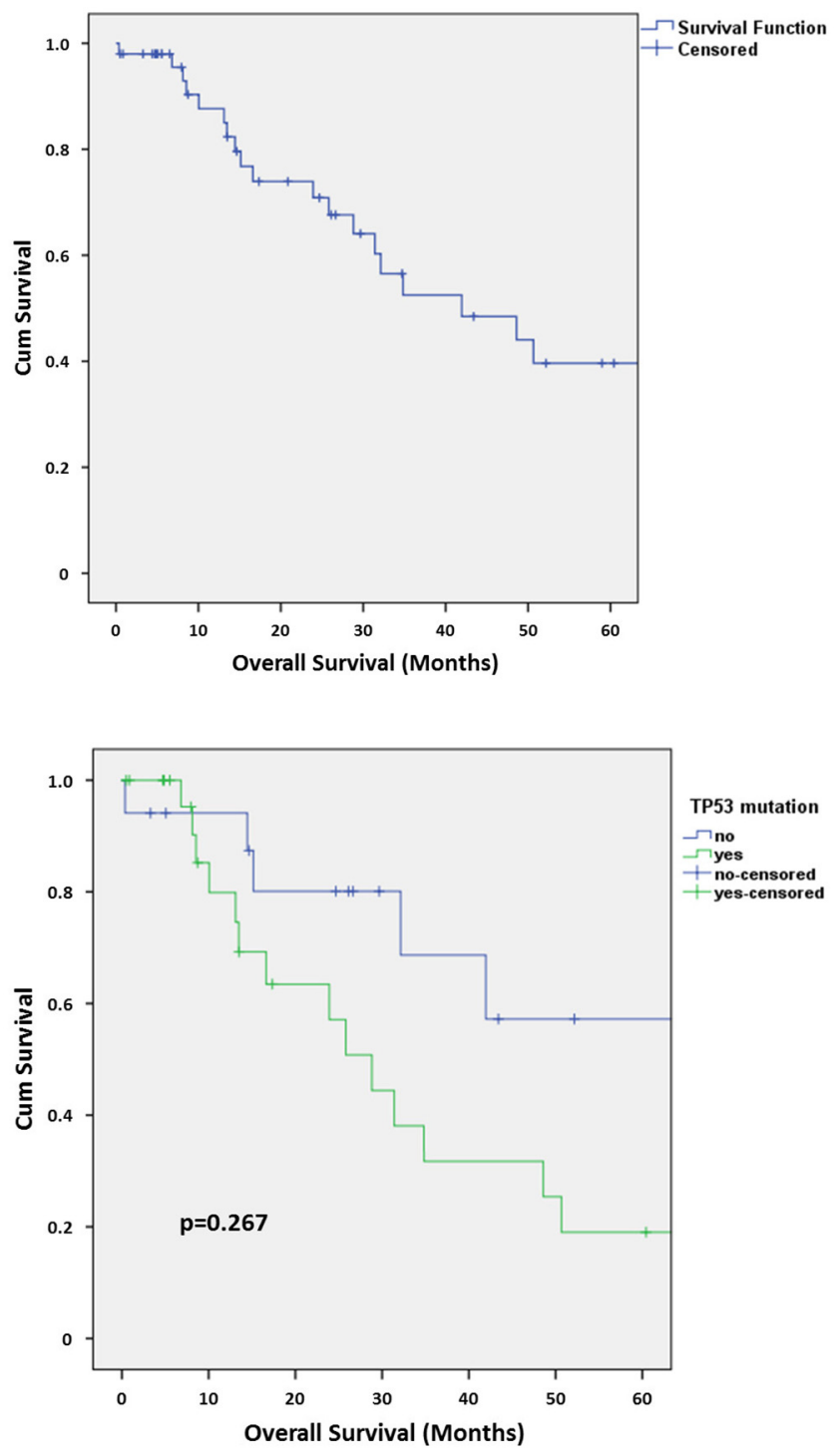

Figure 3: A. Kaplan-Meier chart of overall survival in the collection of SDCs. B. Kaplan-Meier chart of overall survival in the collection of SDCs depending on the presence or absence of a TP53 mutation. 
with our immunohistochemical results and potentially pointing to different characteristics of diagnostically employed antibodies, a previously reported study on five SDC cases demonstrated CDK4 protein expression in four of five cases, accompanied by MDM2 protein expression in two cases; however, $C D K 4$ and $M D M 2$ amplification status was not determined in that study [21].

As described above, in a subset of liposarcomas the small subgroup of MDM2+/CDK4- tumors shows favorable prognostic features compared to MDM2+/ CDK4+ tumors [10]. In our collection of SDCs the 5-year OS was $39.6 \%$. We could not detect any statistically significant differences of the 5-year OS in the MDM2 and/ or $C D K 4$ amplified/grey-zone amplified subgroups, which is probably due to the limited case numbers contained in the smaller subgroups. Larger cohorts of these aggressive tumors should be analyzed for a better understanding of a potential prognostic impact of $M D M 2$ and/or $C D K 4$ alterations.

In this study, we investigated for the first time systematically the involvement of MDM2 and CDK4 in the carcinogenesis of SDCs by analyzing the TP53 mutational status, $M D M 2$ and $C D K 4$ amplification and $H M G A 2$ rearrangement/amplification as well as protein expression of p53, MDM2 and CDK4. We showed that in subgroups of SDCs, $M D M 2$ and/or $C D K 4$ amplification might play a pathogenic role, in part apparently in association with other genetic alterations. Further work is mandatory to clarify the role of MDM2 and CDK4 alterations in the tumorigenesis of SDCs (especially in the TP53 mutated subgroup), the potential prognostic relevance of these alterations in SDCs and the feasibility of MDM2- and/or CDK4-directed therapeutic strategies in SDCs.

\section{MATERIALS AND METHODS}

\section{Patient data and specimens}

The investigation was conducted according to the Declaration of Helsinki on biomedical research involving human subjects. The retrospective study included 51 patients with newly diagnosed salivary duct carcinoma. Among these, 30 cases were derived from the University Hospital of Muenster and 21 cases from the University Hospital of Cologne (the latter having partly been included in a previous study [3]). Patients from Cologne have been treated at the Department of Otorhinolaryngology, Head and Neck Surgery at the University Hospital of Cologne between 1998 and 2011, patients from Muenster have been treated at the Department of Otorhinolaryngology, Head and Neck Surgery at the University Hospital of Muenster between 2000 and 2014. All patients were subjected to primary definitive surgery and potential adjuvant radiation according to patients' cancer stage. Tumor staging was adapted to the $7^{\text {th }}$ edition of the UICC TNM classification for carcinomas of the salivary glands. Patients were followed up at the outpatients department of Cologne or Muenster, respectively, at periodic visits in 3 to 6 months. At the time of analysis, 22 patients had died and 11 patients had developed a histologically confirmed relapse. Mean follow-up time was 22.2 months (range 0 to 161). The study was approved by the local Ethics Committees.

Formalin-fixed and paraffin-embedded (FFPE) material of the patients was obtained from the archives of the Departments of Pathology at the University Hospitals of Cologne and Muenster, respectively. All tumors were re-evaluated microscopically and by means of immunohistochemistry by two experienced pathologists with regard to histopathological diagnosis in accordance with WHO 2005 classification of tumors of salivary glands.

From FFPE material of all included cases, two core biopsies out of the tumor area were taken to assemble tissue microarrays (TMA).

For statistical analysis, the IBM SPSS Statistics 22 software (SPSS Inc., Chicago, IL, USA) was applied and Kaplan-Meier survival analysis and Log rank test were performed. The significance level was set at $\mathrm{p}<0.05$.

\section{FISH analyses and immunohistochemistry}

Fluorescence in-situ hybridization (FISH) analyses and immunohistochemical stainings were conducted on slides from TMAs. FISH analyses were performed as described previously $[22,23]$ using the ZytoLight ${ }^{\circledR}$ SPEC MDM2/CEN 12 Dual Color Probe for assessment of MDM2 amplification and the ZytoLight ${ }^{\circledR}$ SPEC CDK4/CEN 12 Dual Color Probe for assessment of CDK4 amplification (ZytoVision $\mathrm{GmbH}$, Bremerhaven, Germany). HMGA2 FISH analysis was performed according to a previously published assay [16, 24] using BACs RP11-662G15 and RP11-1025D9 (Life Technologies by Thermo Fisher Scientific Inc., Waltham, USA). At least 40-60 tumor cell nuclei of each tumor sample were analyzed. Amplification of MDM2 and $C D K 4$ was defined as an $M D M 2 /$ centromer 12 (CEN12) or $C D K 4 /$ centromer 12 (CEN12) ratio $\geq 2.0$ or an average number of $M D M 2$ or $C D K 4$ signals per tumor cell nucleus $\geq 6$ or large clusters of $M D M 2$ or $C D K 4$ signals $\geq 10 \%$, respectively. Grey-zone amplification was defined as an $M D M 2 /$ centromer $12(\mathrm{CEN} 12)$ or $C D K 4 /$ centromer 12 (CEN12) ratio $\geq 1.8$ or as microclusters of $\geq 5 M D M 2$ or $C D K 4$ signals in $\geq 15 \%$ of tumor cell nuclei, respectively, based on modified scoring algorithms for HER2 and FGFR1 as published before [23, 25]. For HMGA2 rearrangement and amplification were evaluated as published before [16].

Immunohistochemical staining was conducted on a Dako Autostainer (Dako Deutschland GmbH, Hamburg, Germany) following the manufacturer's instructions. Three $\mu \mathrm{m}$ sections were cut from the TMAs, followed by 
heat induced antigen retrieval in low (for p53) or high (for MDM2 and CDK4) $\mathrm{pH}$ buffer. For visualization LSAB method with AP/RED was used. Following antibodies and concentrations were used: p53 (1:3000, clone DO-7, Dako), MDM2 (1:100, clone IF2, Invitrogen by Thermo Fisher Scientific Inc., Waltham, USA) and CDK4 (1:100, clone DCS-31, Invitrogen). Sections were counterstained with hematoxylin and tiled with Cytoseal (Thermo Fisher Scientific Inc.). For scoring of the immunohistochemical stainings for p53, MDM2 and CDK4 only nuclear staining was rated. The staining intensity was evaluated semiquantitavely into $0,1,2$ or 3 by comparing within the different tumor samples. To determine percentage labelling indices, all tumor cells within the cores were analyzed using high-power (400x) magnification. A sum score was calculated out of the staining intensity and the percentage labelling index, and for MDM2 and CDK4 value ranges for staining level were defined as follows: 0 : no staining; $>0$ to 50 : low staining level; $>50$ to 100 : intermediate staining level; $>100$ : strong staining. Sum score of p53 staining was interpreted modified according to Boyle et al. [26] as follows: 0: extreme negative (EN); $>50$ : extreme positive (EP); $>0$ to 50 : non-extreme (NE, intermediate patterns).

\section{Assessment of TP53 mutational status}

To complete the data on TP53 mutational status as previously described for a smaller subset of samples [3] for the whole cohort, assessment of TP53 mutational status was carried out as follows:

\section{Tumor macrodissection, DNA extraction and quantification}

Sections were prepared from FFPE material and stained with hematoxylin \& eosin (H\&E). Six additional sections of $6 \mu \mathrm{m}$ thickness were cut, mounted onto glass slides and used for macrodissection. In total, $1 \mathrm{~cm}^{2}$ tumor area corresponding to the tumor area of H\&E-stained section was scraped off with a scalpel and collected into plastic tubes. Subsequently, the DNA was automatically extracted using the Maxwell DNA FFPE isolation kit on a Maxwell platform (Promega $\mathrm{GmbH}$, Mannheim, Germany). Fluorometric DNA quantification was performed according to the Qubit dsDNA HS assay (Qubit 2.0, Life Technologies, Carlsbad, CA, USA).

\section{NGS library construction}

Pre-verified multiplex PCR primer sets (summarized in Supporting Information Supplemental Table 1 A) were used to amplify the exonic region of TP53 (customized GeneRead DNAseq Mix-n-Match V2 panel, Qiagen GmbH, Hilden, Germany). Target enrichment was processed by means of the GeneRead DNAseq Panel PCR V2 Kit (Qiagen), following the manufacturer's instructions. All purification and size selection steps were performed utilizing Agencourt AMPure XP magnetic beads (Beckman Coulter, Inc., Brea, CA, USA). End repair, A-addition and ligation to NEXTflex-96 DNA barcodes (Bioo Scientific, Austin, Texas, USA) was carried out using the GeneRead DNA Library I Core Kit (Qiagen). Amplification of adapter-ligated DNA was conducted using NEXTflex primers (Bioo Scientific) and the HiFi PCR Master Mix (GeneRead DNA I Amp Kit, Qiagen). Next generation sequencing was performed applying $12.5 \mathrm{pM}$ library pools (2\% PhiX V3 control) and the MiSeq Reagent v2 chemistry (Illumina, Inc., San Diego, Ca, USA).

\section{NGS data analysis}

Fastq files were generated by the MiSeq Reporter software (Illumina) and further analyzed by means of the CLC Biomedical Genomics Workbench software (CLC bio, Qiagen). The total batch of identified TP53 variants was filtered according to following criteria and then validated by Sanger sequencing: Hotspot artefacts and reading errors were filtered, which are recognized by high occurrence and constant frequency within the cohort. In addition, synonymous variants, germline single nucleotide polymorphisms (SNPs) and all variants below 4\% allelic frequency were filtered, ending up with variants listed in Supporting Information Supplemental Table 2. Additional information on the functional impact of detected TP53 mutations is provided in Supporting Information Supplemental Table 3.

\section{Sanger sequencing}

Conventional Sanger sequencing was conducted according to standard procedures using the BigDye Terminator v3.1 Cycle Sequencing Kit (Thermo Fisher Scientific Inc.) and various TP53 primer sets (summarized in Supporting Information Supplemental Table 1 B).

\section{ACKNOWLEDGMENTS}

There are no financial disclosures from any authors.

\section{CONFLICTS OF INTEREST}

The authors disclose no potential conflicts of interest.

\section{REFERENCES}

1. Eveson JW, Auclair P, Gnepp DR, El-Naggar AK. (2005). Tumours of the Salivary glands. In: Barnes L, Eveson JW, 
Reichart P, Sidransky D, eds. World health organization classification of tumours Pathology and genetics Head and neck tumours. (Lyon: IARC Press), pp. 209-81.

2. Chiosea SI, Williams L, Griffith CC, Thompson LD, Weinreb I, Bauman JE, Luvison A, Roy S, Seethala RR, Nikiforova MN. Molecular characterization of apocrine salivary duct carcinoma. The American Journal of Surgical Pathology. 2015; 39: 744-52. doi: 10.1097/ PAS.0000000000000410.

3. Grunewald I, Vollbrecht C, Meinrath J, Meyer MF, Heukamp LC, Drebber U, Quaas A, Beutner D, Huttenbrink KB, Wardelmann E, Hartmann W, Buttner R, Odenthal M, et al. Targeted next generation sequencing of parotid gland cancer uncovers genetic heterogeneity. Oncotarget. 2015; 6: 18224-37. doi: 10.18632/oncotarget.4015.

4. Wade M, Li YC, Wahl GM. MDM2, MDMX and p53 in oncogenesis and cancer therapy. Nature reviews Cancer. 2013; 13: 83-96. doi: 10.1038/nrc3430.

5. Vassilev LT, Vu BT, Graves B, Carvajal D, Podlaski F, Filipovic Z, Kong N, Kammlott U, Lukacs C, Klein C, Fotouhi N, Liu EA. In vivo activation of the p53 pathway by small-molecule antagonists of MDM2. Science (New York, NY). 2004; 303: 844-8. doi: 10.1126/science. 1092472.

6. Ray-Coquard I, Blay JY, Italiano A, Le Cesne A, Penel N, Zhi J, Heil F, Rueger R, Graves B, Ding M, Geho D, Middleton SA, Vassilev LT, et al. Effect of the MDM2 antagonist RG7112 on the P53 pathway in patients with MDM2-amplified, well-differentiated or dedifferentiated liposarcoma: an exploratory proof-of-mechanism study. The Lancet Oncology. 2012; 13: 1133-40. doi: 10.1016/ S1470-2045(12)70474-6.

7. Persson F, Andren Y, Winnes M, Wedell B, Nordkvist A, Gudnadottir G, Dahlenfors R, Sjogren H, Mark J, Stenman G. High-resolution genomic profiling of adenomas and carcinomas of the salivary glands reveals amplification, rearrangement, and fusion of HMGA2. Genes, chromosomes \& cancer. 2009; 48: 69-82. doi: 10.1002/ gcc.20619.

8. Lundgren K, Montes de Oca Luna R, McNeill YB, Emerick EP, Spencer B, Barfield CR, Lozano G, Rosenberg MP, Finlay CA. Targeted expression of MDM2 uncouples $\mathrm{S}$ phase from mitosis and inhibits mammary gland development independent of p53. Genes \& development. 1997; 11: 714-25.

9. Italiano A, Bianchini L, Keslair F, Bonnafous S, CardotLeccia N, Coindre JM, Dumollard JM, Hofman P, Leroux A, Mainguene C, Peyrottes I, Ranchere-Vince D, Terrier P, et al. HMGA2 is the partner of MDM2 in well-differentiated and dedifferentiated liposarcomas whereas CDK4 belongs to a distinct inconsistent amplicon. International journal of cancer. 2008; 122: 2233-41. doi: 10.1002/ijc.23380.

10. Italiano A, Bianchini L, Gjernes E, Keslair F, RanchereVince D, Dumollard JM, Haudebourg J, Leroux A, Mainguene C, Terrier P, Chibon F, Coindre JM, Pedeutour F.
Clinical and biological significance of CDK4 amplification in well-differentiated and dedifferentiated liposarcomas. Clinical cancer research. 2009; 15: 5696-703. doi: 10.1158/1078-0432.CCR-08-3185.

11. Dickson MA. Molecular pathways: CDK4 inhibitors for cancer therapy. Clinical cancer research. 2014; 20: 337983. doi: 10.1158/1078-0432.CCR-13-1551.

12. Leontieva OV, Blagosklonny MV. CDK4/6-inhibiting drug substitutes for $\mathrm{p} 21$ and $\mathrm{p} 16$ in senescence: duration of cell cycle arrest and MTOR activity determine geroconversion. Cell Cycle. 2013; 12: 3063-9. doi: 10.4161/cc.26130.

13. Ashar HR, Fejzo MS, Tkachenko A, Zhou X, Fletcher JA, Weremowicz S, Morton CC, Chada K. Disruption of the architectural factor HMGI-C: DNA-binding AT hook motifs fused in lipomas to distinct transcriptional regulatory domains. Cell. 1995; 82: 57-65.

14. Schoenmakers EF, Wanschura S, Mols R, Bullerdiek J, Van den Berghe H, Van de Ven WJ. Recurrent rearrangements in the high mobility group protein gene, HMGI-C, in benign mesenchymal tumours. Nat Genet. 1995; 10: 436-44. doi: 10.1038/ng0895-436.

15. Berner JM, Meza-Zepeda LA, Kools PF, Forus A, Schoenmakers EF, Van de Ven WJ, Fodstad O, Myklebost O. HMGIC, the gene for an architectural transcription factor, is amplified and rearranged in a subset of human sarcomas. Oncogene. 1997; 14: 2935-41. doi: 10.1038/ sj.onc. 1201135.

16. Bahrami A, Perez-Ordonez B, Dalton JD, Weinreb I. An analysis of PLAG1 and HMGA2 rearrangements in salivary duct carcinoma and examination of the role of precursor lesions. Histopathology. 2013; 63: 250-62. doi: 10.1111/ his. 12152 .

17. Alkhalaf M, Ganguli G, Messaddeq N, Le Meur M, Wasylyk B. MDM2 overexpression generates a skin phenotype in both wild type and p53 null mice. Oncogene. 1999; 18: 1419-34. doi: 10.1038/sj.onc.1202448.

18. Jones SN, Hancock AR, Vogel H, Donehower LA, Bradley A. Overexpression of $\mathrm{Mdm} 2$ in mice reveals a p53independent role for $\mathrm{Mdm} 2$ in tumorigenesis. Proceedings of the National Academy of Sciences of the United States of America. 1998; 95: 15608-12.

19. Cheng X, Xia W, Yang JY, Hsu JL, Lang JY, Chou CK, Du Y, Sun HL, Wyszomierski SL, Mills GB, Muller WJ, Yu D, Hung MC. Activation of murine double minute 2 by Akt in mammary epithelium delays mammary involution and accelerates mammary tumorigenesis. Cancer research. 2010; 70: 7684-9. doi: 10.1158/0008-5472.CAN-09-3231.

20. Limesand KH, Schwertfeger KL, Anderson SM. MDM2 is required for suppression of apoptosis by activated Akt1 in salivary acinar cells. Molecular and cellular biology. 2006; 26: 8840-56. doi: MCB.01846-05 [pii].

21. Etges A, Pinto DS, Jr., Kowalski LP, Soares FA, Araujo VC. Salivary duct carcinoma: immunohistochemical profile of 
an aggressive salivary gland tumour. Journal of clinical pathology. 2003; 56: 914-8.

22. Friedrichs N, Kriegl L, Poremba C, Schaefer KL, Gabbert HE, Shimomura A, Paggen E, Merkelbach-Bruse S, Buettner R. Pitfalls in the detection of $\mathrm{t}(11 ; 22)$ translocation by fluorescence in situ hybridization and RT-PCR: a single-blinded study. Diagnostic molecular pathology: the American journal of surgical pathology, part B. 2006; 15: 83-9. doi: 00019606-200606000-00004 [pii].

23. Schildhaus HU, Heukamp LC, Merkelbach-Bruse S, Riesner K, Schmitz K, Binot E, Paggen E, Albus K, Schulte W, Ko YD, Schlesinger A, Ansen S, EngelRiedel W, et al. Definition of a fluorescence in-situ hybridization score identifies high- and low-level FGFR1 amplification types in squamous cell lung cancer. Modern pathology. 2012; 25: 1473-80. doi: 10.1038/ modpathol.2012.102.
24. Bahrami A, Dalton JD, Shivakumar B, Krane JF. PLAG1 alteration in carcinoma ex pleomorphic adenoma: immunohistochemical and fluorescence in situ hybridization studies of 22 cases. Head Neck Pathol. 2012; 6: 328-35. doi: 10.1007/s12105-012-0353-8.

25. Wolff AC, Hammond ME, Schwartz JN, Hagerty KL, Allred DC, Cote RJ, Dowsett M, Fitzgibbons PL, Hanna WM, Langer A, McShane LM, Paik S, Pegram MD, et al. American Society of Clinical Oncology/College of American Pathologists guideline recommendations for human epidermal growth factor receptor 2 testing in breast cancer. Journal of clinical oncology. 2007; 25: 118-45. doi: JCO.2006.09.2775 [pii].

26. Boyle DP, McArt DG, Irwin G, Wilhelm-Benartzi CS, Lioe TF, Sebastian E, McQuaid S, Hamilton PW, James JA, Mullan PB, Catherwood MA, Harkin DP, Salto-Tellez M. The prognostic significance of the aberrant extremes of p53 immunophenotypes in breast cancer. Histopathology. 2014; 65: 340-52. doi: 10.1111/his.12398. 